\title{
AN OPTIMAL OPERATING STRATEGY FOR FED-BATCH FERMENTATIONS BY FEEDING THE OVERFLOW METABOLITE
}

\author{
S. Valentinotti *, C. Cannizzaro *, B. Srinivasan ${ }^{* *}$, \\ D. Bonvin ${ }^{* *, 1}$ \\ * Laboratoire de Génie Chimique et Biologique \\ ** Laboratoire d'Automatique \\ Ecole Polytechnique Fédérale de Lausanne \\ CH - 1015 Lausanne, Switzerland
}

\begin{abstract}
Optimization of the fed-batch fermentation of Saccharomyces cerevisiae is analyzed. Due to the limited oxygen uptake capacity of the cells, the overflow metabolite ethanol is formed when the substrate concentration is above some critical value. This value decreases during the course of an experiment due to the reduction in dissolved oxygen concentration resulting from biomass formation. Optimal operation corresponds to regulating the substrate concentration along this time-varying critical value. This paper proposes a novel strategy to implement this optimal solution, whereby ethanol is fed along with the substrate and its concentration in the reactor regulated around the inlet concentration value. Suboptimal strategies of practical interest are also discussed and simulation results are presented.
\end{abstract}

Keywords: Fed-batch fermentation, Overflow metabolite, Bottleneck principle, Optimization, Ethanol regulation.

\section{INTRODUCTION}

Biotechnology has risen to becoming one of the active research areas in the control community. In this work, the optimization of a key biotechnological process, the production of baker's yeast, is studied. Though presented for baker's yeast, the results are generally applicable to fermentation processes with microorganisms that present an overflow metabolism.

Numerous models have been proposed to describe the behavior of Saccharomyces cerevisiae under different growth conditions (Nielsen and Villadsen, 1994). The model used in this work was proposed by (Sonnleitner and Käppeli, 1986). It as-

\footnotetext{
1 Corresponding author: dominique.bonvin@epfl.ch
}

sumes that the oxidative capacity of $S$. cerevisiae is limited and constitutes a bottleneck in the oxidative metabolism. The size of this bottleneck may change from experiment to experiment and even during a given experiment due to changes in the cell metabolism, nutrient limitation, or other factors (van Hoek et al., 1998). When the substrate uptake rate exceeds the oxidative capacity, the overflow metabolite ethanol is formed.

Maximization of biomass production is obtained when the glucose flux exactly matches the oxidative capacity of the cells. However, industrial bioreactors are often operated at substrate concentrations well under this critical value in order to avoid either yield losses when substrate is transformed into ethanol or accumulation of the overflow metabolite that might be toxic. This work 
proposes a methodology for ensuring optimality by operating the reactor at or near this unknown, time-varying critical value.

Regulating the concentration of the overflow metabolite has been used for the purpose of optimization in several works (Axelsson, 1989; Chen et al., 1995; Valentinotti et al., 2003). However, true optimality would require regulating the ethanol concentration at zero, which is not possible due to the non-zero resolution of the ethanol sensor. Thus, those approaches are at best sub-optimal.

In this work, the non-intuitive idea of adding the overflow metabolite in the feed stream is used. By choosing the ethanol regulation set point equal to its concentration in the feed, optimal operation can be achieved. The main advantage is that the sensor resolution is no longer a critical issue. Furthermore, if desired, sub-optimal operation can be obtained by adjusting the ethanol set point relative to its concentration in the feed.

The paper is organized as follows. In Section 2, a macroscopic process model is presented. Section 3 formulates the optimization problem and its nominal solution, while Section 4 discusses three on-line operating strategies. The adaptive control strategy is presented in Section 5 and simulation results are shown in Section 6.

\section{PROCESS MODELING}

A macroscopic description of the metabolism of $S$. cerevisiae fermentation includes the following reactions:

$$
\begin{aligned}
& S+a_{1} O_{2} \stackrel{r_{1}}{\rightarrow} b_{1} X+c_{1} C O_{2} \\
& S_{r_{2}}^{\rightarrow} b_{2} X+c_{2} C O_{2}+d_{2} P \\
& P+a_{3} O_{2} \stackrel{r_{3}}{\rightarrow} b_{3} X+c_{3} C O_{2}
\end{aligned}
$$

where $S$ is the substrate, $P$ the reaction product ethanol that can also be oxidized by the cells, $\mathrm{X}$ the biomass, and $\mathrm{CO}_{2}$ and $\mathrm{O}_{2}$ carbon dioxide and oxygen, respectively. $a_{i}, b_{i}, c_{i}, d_{i}$ and $r_{i}$ are the yield coefficients and the reaction rate of the $i^{t h}$ reaction, respectively.

In this work, the overflow metabolism (bottleneck) model proposed by (Sonnleitner and Käppeli, 1986) is used. It assumes a limited respiratory capacity of the cells. The uptake of the glucose fed to the reactor is assumed to occur at the following rate:

$$
r_{s}=k_{s} \frac{S}{S+K_{s}} \quad\left[\frac{g \text { of } S}{g \text { of } X h}\right]
$$

The rate at which the cells can oxidize the substrate is given by :

$$
r_{o}=k_{o} \frac{O_{2}}{O_{2}+K_{o}} \quad\left[\frac{g \text { of } O_{2}}{g \text { of } X h}\right]
$$

The rate $r_{o}$ is seen as the bottleneck since it limits the amount of glucose that can be oxidized. Thus, Reaction (1) takes place as long as sufficient glucose and oxygen are available in the reactor. Its rate is determined by the smallest of the rates at which glucose and oxygen are taken up by the cells, $r_{s}$ and $r_{o} / a_{1}$, respectively:

$$
r_{1}=\min \left(r_{s}, \frac{r_{o}}{a_{1}}\right)
$$

The glucose concentration at which the oxidative capacity saturates is defined as $S_{\text {crit }}$, for which $r_{s}=r_{o} / a_{1}$. It follows that $S_{c r i t}=r_{o} K_{S} /\left(a_{1} k_{s}-\right.$ $\left.r_{o}\right)$ is a function of the dissolved oxygen concentration $\mathrm{O}_{2}$. When the glucose flux is too large to fit through the bottleneck, i.e. $r_{s}>r_{o} / a_{1}$ corresponding to $S>S_{\text {crit }}$, the excess will overflow into the reductive metabolism resulting in ethanol production according to Reaction (2). This is in fact what gives this metabolism its name. The rate at which this reaction takes place is given by:

$$
r_{2}=\max \left(0, r_{s}-\frac{r_{o}}{a_{1}}\right)
$$

If the glucose flux does not use up the whole oxidative capacity of the cells, the ethanol present in the reactor is oxidized simultaneously via Reaction (3). The excess oxidative capacity is given by $r_{o}-a_{1} r_{s}$, and the rate at which ethanol is oxidized is therefore:

$$
\begin{gathered}
r_{3}=\max \left(0, \min \left(r_{p}, \frac{r_{o}-a_{1} r_{s}}{a_{3}}\right)\right) \\
r_{p}=k_{p} \frac{P}{P+K_{p}} \quad\left[\frac{g \text { of } P}{g \text { of } X h}\right]
\end{gathered}
$$

Based on the reaction model (1)-(3), the following macroscopic mass balances can be derived:

$$
\begin{aligned}
\frac{d(V X)}{d t} & =\left(b_{1} r_{1}+b_{2} r_{2}+b_{3} r_{3}\right) V X \\
\frac{d(V S)}{d t} & =-\left(r_{1}+r_{2}\right) V X+F S_{i n} \\
\frac{d(V P)}{d t} & =\left(d_{2} r_{2}-r_{3}\right) V X+F P_{i n} \\
\frac{d\left(V O_{2}\right)}{d t} & =k_{L} a V\left(O_{2}^{*}-O_{2}\right)-\left(a_{1} r_{1}+a_{3} r_{3}\right) V X \\
\frac{d V}{d t} & =F
\end{aligned}
$$

where $F$ is the substrate feed rate, $V$ the volume, and $S_{\text {in }}$ and $P_{\text {in }}$ the inlet concentrations of $S$ and $P$, respectively. The dissolved oxygen concentration in the bioreactor is given by (13), where $k_{L} a$ is the overall mass transfer coefficient, and $O_{2}^{*}$ the dissolved oxygen equilibrium concentration. For simplicity, it is assumed that $k_{L} a$ and $O_{2}^{*}$ remain constant throughout the experiment. 
The model parameters are given in Tables 1 and 2 , while the operating and initial conditions used in the simulation are provided in Table 3 .

\begin{tabular}{|lll|}
\hline Parameter & Value & Unit \\
\hline$a_{1}$ & 0.396 & g of $O_{2} / \mathrm{g}$ of $S$ \\
$b_{1}$ & 0.490 & g of $X / \mathrm{g}$ of $S$ \\
$c_{1}$ & 0.590 & g of $C O_{2} / \mathrm{g}$ of $S$ \\
$b_{2}$ & 0.050 & g of $X / \mathrm{g}$ of $S$ \\
$c_{2}$ & 0.462 & g of $C O_{2} / \mathrm{g}$ of $S$ \\
$d_{2}$ & 0.480 & g of $P / \mathrm{g}$ of $S$ \\
$a_{3}$ & 1.104 & g of $O_{2} / \mathrm{g}$ of $P$ \\
$b_{3}$ & 0.720 & g of $X / \mathrm{g}$ of $P$ \\
$c_{3}$ & 0.625 & g of $C O_{2} / \mathrm{g}$ of $P$ \\
\hline
\end{tabular}

Table 1. Yield coefficients for the proposed reaction mechanism.

\begin{tabular}{|lll|}
\hline Parameter & Value & Unit \\
\hline$k_{s}$ & 3.500 & $\mathrm{~g}$ of $S / \mathrm{g}$ of $X \mathrm{~h}$ \\
$k_{o}$ & 0.256 & $\mathrm{~g}$ of $O_{2} / \mathrm{g}$ of $X \mathrm{~h}$ \\
$k_{p}$ & 0.170 & $\mathrm{~g}$ of $P / \mathrm{g}$ of $X \mathrm{~h}$ \\
$K_{s}$ & 0.100 & $\mathrm{~g}$ of $S / 1$ \\
$K_{o}$ & 0.001 & $\mathrm{~g}$ of $O_{2} / 1$ \\
$K_{p}$ & 0.100 & $\mathrm{~g}$ of $P / 1$ \\
\hline
\end{tabular}

Table 2. Kinetic parameters for the rates $r_{s}, r_{o}$, and $r_{p}$.

\begin{tabular}{|lll|}
\hline Variable & Value & Unit \\
\hline$S_{i n}$ & 300 & $\mathrm{~g} / 1$ \\
$P_{i n}$ & 10 & $\mathrm{~g} / 1$ \\
$O_{2}^{*}$ & 0.039 & $\mathrm{~g} / 1$ \\
$k_{L} a$ & 250 & $h^{-1}$ \\
$V_{\max }$ & 8 & 1 \\
$F_{\max }$ & 3 & $\mathrm{l} / \mathrm{h}$ \\
\hline$X_{o}$ & 1.5 & $\mathrm{~g} / 1$ \\
$S_{o}$ & 0.023 & $\mathrm{~g} / 1$ \\
$P_{o}$ & 10 & $\mathrm{~g} / 1$ \\
$O_{2}$ & 0.039 & $\mathrm{~g} / 1$ \\
$V_{o}$ & 4 & 1 \\
\hline
\end{tabular}

Table 3. Operating and initial conditions

\section{OPTIMIZATION PROBLEM AND NOMINAL SOLUTION}

From a practitioner's perspective, the goal is to maximize the amount of biomass with minimum batch time, which in fact are two objectives in one. Thus, from an optimization perspective, these two objectives need to be combined. In this paper, the batch time is considered as the cost function to be minimized, and the biomass productivity as a constraint to be met. As a result, the optimization problem is formulated as follows: given operational constraints, determine the feeding strategy that minimizes the batch time while ensuring that the amount of biomass at final time is at least the prescribed quantity $(V X)_{d e s}$ :

$$
\min _{t_{f}, F(t)} J=t_{f}
$$

subject to $(10)-(14)$

$$
\begin{aligned}
& 0 \leq F(t) \leq F_{\max } \\
& V(t) \leq V_{\max }, V\left(t_{f}\right) X\left(t_{f}\right) \geq(V X)_{\text {des }}
\end{aligned}
$$

where $t_{f}$ is the final time, $V_{\max }$ the maximal volume, $F_{\max }$ the maximum feed rate at which the substrate can be fed, and $(V X)_{\text {des }}$ the desired minimal amount of biomass computed as:

$$
(V X)_{d e s}=V_{o} X_{o}+b_{1} S_{i n}\left(V_{\max }-V_{o}\right)
$$

which corresponds to the amount of biomass that can be attained from the substrate. Note that, due to the presence of ethanol in the feed, it is possible to produce slightly more biomass than $(V X)_{\text {des }}$. The optimal solution of (15) obtained numerically is shown in Figure 1.

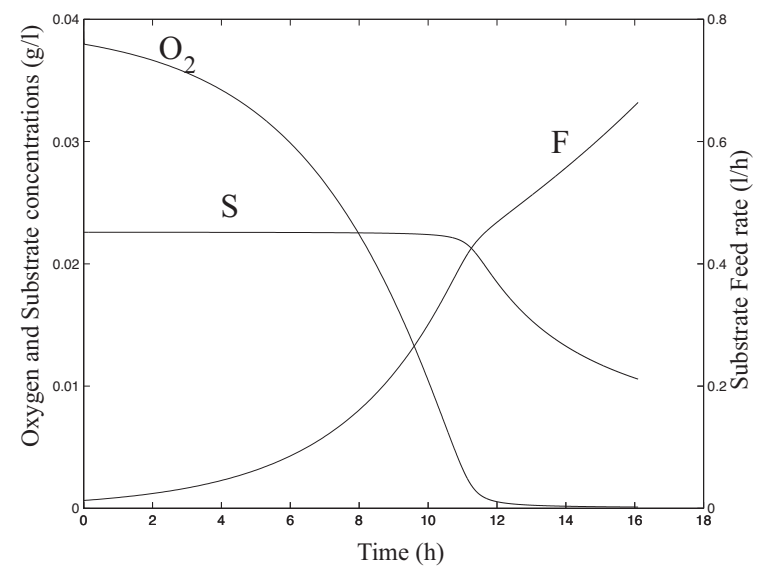

Fig. 1. Optimal feed rate profile and evolutions of oxygen and substrate concentrations.

It has been argued in Sonnleitner and Käppeli (1986) that exactly filling the bottleneck is optimal in some sense. Here, it will be shown that, for the optimization problem (15), the optimal solution in fact corresponds to exactly filling the bottleneck, i.e. regulating $S$ at $S_{\text {crit }}$. To arrive at this conclusion, the two cases with $S \geq S_{\text {crit }}$ and $S \leq S_{\text {crit }}$ are considered, and it follows that $S=S_{\text {crit }}$ is indeed optimal.

- For $S \geq S_{\text {crit }}$, biomass is produced from the substrate by Reactions 1 and 2, and eventually the overflown ethanol is converted to biomass through Reaction 3. Thus, for the consumption of one unit of substrate, the quantity of biomass produced is $\left(b_{1} \alpha+\right.$ $\left.\left(b_{2}+d_{2} b_{3}\right)(1-\alpha)\right)$, where $\alpha=r_{1} / r_{s}, 0 \leq$ $\alpha \leq 1$. Since $b_{1}>\left(b_{2}+d_{2} b_{3}\right)$, the maximum corresponds to $\alpha=1$, i.e. $r_{1}=r_{s}$ where the bottleneck is exactly filled. So, for $S>S_{\text {crit }}$, the desired productivity cannot be achieved with the substrate alone, and some of the ethanol in the feed stream must be consumed in order to produce the difference in the desired biomass production. 
- For $S \leq S_{\text {crit }}$, there is space in the bottleneck for some of the ethanol in the inlet to be converted to biomass, i.e. $r_{3}=\left(r_{o}-a_{1} r_{1}\right) / a_{3}$. So, the rate of production of biomass is: $\left(\frac{b_{1}}{a_{1}} \beta+\frac{b_{3}}{a_{3}}(1-\beta)\right) r_{o} X V$, where $\beta=a_{1} r_{1} / r_{o}$, $0 \leq \beta \leq 1$. Since, $\frac{b_{1}}{a_{1}}>\frac{b_{3}}{a_{3}}$, the maximum value is for $\beta=1$, i.e. $r_{1} \stackrel{a_{3}}{=} r_{o} / a_{1}$. In other words, the bottleneck should be entirely filled with substrate in order to minimize time, though the desired productivity could be achieved even by partially filling it.

For the initial condition $S(0)=S_{\text {crit }}\left(O_{2}(0)\right)$, the optimal input $F^{*}$ that enforces $S=S_{\text {crit }}$ can be obtained by differentiating $r_{s}=r_{o} / a_{1}$ once with respect to time:

$$
F^{*}=\left.V \frac{N}{D}\right|_{S=S_{\text {crit }}}
$$

where

$N=a_{1} K_{o} k_{s} S^{2}\left(k_{L} a\left(O_{2}^{*}-O_{2}\right)-r_{o} X\right)+K_{s} k_{o} O_{2}^{2} r_{s} X$

$D=a_{1} K_{o} k_{s} S^{2} O_{2}+K_{s} k_{o} O_{2}^{2}\left(S_{i n}-S\right)$

The first part of the feed rate profile is nearly exponential when oxygen is not limiting, while the second part is almost linear when oxygen limitation occurs after about $10 \mathrm{~h}$. The optimal solution is $t_{f}^{*}=16.12 \mathrm{~h}$. Figure 1 also indicates that $S_{\text {crit }}$ reduces with time.

\section{ON-LINE OPERATING STRATEGIES}

Since the model parameters might not be accurately known and can vary during the batch, the feed rate expression (17) cannot be used to implement the optimal strategy. Instead, it is possible to use the ethanol concentration measurement $P$ to adjust the substrate feed rate $F$.

As seen in the previous section, optimality requires $r_{2}=r_{3}=0$, i.e. neither production nor consumption of ethanol. One possibility is to track the amount of ethanol $V(t) P(t)=$ $(V P)_{\text {ref }}$ (Valentinotti et al., 2003). Another possibility, which involves tracking the concentration of ethanol with $P_{\text {in }} \neq 0$, is discussed next.

Application of the chain rule of differentiation to (12) gives:

$$
\frac{d P}{d t}=\left(d_{2} r_{2}-r_{3}\right) X+\frac{F}{V}\left(P_{\text {in }}-P\right)
$$

Assume that $P_{i n}$ is constant and the ethanol concentration is regulated around the value $P_{r e f}$. If $P(t)=P_{\text {ref }}=P_{i n}$, then $d P / d t=0$ implies $\left(d_{2} r_{2}-r_{3}\right)=0$. However, since $r_{2}$ and $r_{3}$ are nonnegative and cannot be positive simultaneously, $r_{2}=r_{3}=0$.
In addition, depending on the relative values of $P_{\text {ref }}$ and $P_{i n}$, sub-optimal solutions are possible:

- $P_{r e f}<P_{i n}$ (for $r_{2}=0$ and $r_{3}>0$ ): Ethanol is constantly consumed and $S<S_{\text {crit }}$.

- $P_{\text {ref }}>P_{\text {in }}$ (for $r_{2}>0$ and $r_{3}=0$ ): Ethanol is constantly produced and $S>S_{\text {crit }}$.

The larger the difference $\left|P_{r e f}-P_{i n}\right|$, the more sub-optimal the operation will be.

Though it is preferable to keep the operation optimal, there might be biological reasons for choosing sub-optimal operation. Consider the optimal case where the ethanol concentration is regulated at $P_{i n}$. Then, for any corrective action needed, for example, to reject a perturbation, the system has to switch from oxidative to reductive metabolism and vice-versa. In other words, if excess ethanol is produced, some space needs to be created in the bottleneck for it to be consumed. In contrast, this change of metabolism need not take place in suboptimal strategies. Among the two sub-optimal strategies, $P_{\text {ref }}<P_{\text {in }}$ leads to $S<S_{\text {crit }}$, implying that maximal yield is still achieved, but the batch time is longer. On the other hand, $P_{\text {ref }}>P_{\text {in }}$ leads to shorter batch times at the cost of a reduction in yield.

The particular case $P_{i n}=0$ was considered in (Valentinotti, 2001). There, $P_{\text {ref }}$ had to be as low as possible in order to be nearly optimal. Thus, $P_{\text {ref }}$ was chosen based on the resolution of the ethanol sensor, which is no longer the case when a non-zero $P_{i n}$ is used. Furthermore, with $P_{i n}=0$, it is only possible to control the system in the overflow situation since negative concentrations cannot be measured. In contrast, with a non-zero $P_{i n}$, the reference is shifted up to $P_{i n}$ and the system becomes observable and controllable for all three cases - overflow, critical, and underflow.

\section{CONTROLLER DESIGN}

In this section, a linear adaptive controller will be used to maintain the ethanol concentration $P$ constant. Thus, the computation of a linear model will be discussed first, followed by the design of the adaptive controller.

\subsection{Linear model}

The bioreactor is a fed-batch process with no steady-state operating point. However, for optimal operation, $P(t)=P_{i n}$ and $S(t)=S_{\text {crit }}$. Hence, linearization will be performed around these optimal values for $P$ and $S$ while using averaged values for the others, e.g. $\bar{V}$ and $\bar{F}$. 
In order to derive a linear model, it is assumed that $(V S)$ is at quasi-steady state:

$$
\frac{d(V S)}{d t}=-\left(r_{1}+r_{2}\right) X V+F S_{i n}=0
$$

The linearized dynamics will be different depending on whether the second or the third reaction takes place in addition to $r_{1}$. Thus, two cases need to be considered:

- Case A: $r_{2} \neq 0, r_{3}=0$. Here, $r_{1}=r_{o} / a_{1}$ and, from (19), $r_{2} X=(F / V) S_{i n}-\left(r_{o} / a_{1}\right) X$. Using this expression in (18) leads to:

$$
\frac{d P}{d t}=\frac{F}{V}\left(d_{2} S_{i n}+P_{i n}-P\right)-\frac{d_{2} r_{o} X}{a_{1}}(20)
$$

or, in linearized form:

$$
\frac{d P}{d t}=-\alpha P+\beta_{a} F-\alpha \beta_{a} V-\gamma_{a} X
$$

with $\alpha=\bar{F} / \bar{V}, \beta_{a}=\left(d_{2} S_{\text {in }}+P_{\text {in }}-P_{\text {ref }}\right) / \bar{V}$ and $\gamma_{a}=d_{2} \bar{r}_{o} / a_{1}$.

Since $V$ depends on $F$ according to (14), (21) leads to the following transfer function model:

$$
P(s)=\frac{\beta_{a}(s-\alpha)}{s(s+\alpha)}\left[F(s)-W_{a}(s)\right]
$$

where $W_{a}(s)=\frac{\gamma_{a} s}{\beta_{a}(s-\alpha)} X(s)$ represents the equivalent flowrate that is needed for biomass growth and, as such, can be seen as an input perturbation to the transfer between $F$ and $P$. The corresponding discrete-time model reads:

$$
P(k h)=\frac{B_{a}\left(q^{-1}\right)}{A\left(q^{-1}\right)}\left[F(k h)-w_{a}(k h)\right]
$$

where, with the $\mathrm{ZOH}$ approximation, $A\left(q^{-1}\right)=$ $\left(1-q^{-1}\right)\left(1-e^{-\alpha h} q^{-1}\right), B_{a}\left(q^{-1}\right)=\left(\delta_{a}-\right.$ $\left.\beta_{a} h\right) q^{-1}-\left(\delta_{a}-\beta_{a} h e^{-\alpha h}\right) q^{-2}$ and $w_{a}(k h)=$ $e^{\alpha h} w_{a}(k h-h)+\left(\gamma_{a} / \beta_{a}\right)[X(k h)-X(k h-h)]$, with $\delta_{a}=2 \beta_{a}\left(1-e^{-\alpha h}\right) / \alpha-\beta_{a} h, h$ the sampling period, $k h$ the sampling instant, and $q^{-1}$ the backward-shift operator.

- Case B: $r_{2}=0, r_{3} \neq 0$. Here, $r_{1}=r_{s}$ and, from (19), $r_{1} X=(F / V) S_{i n}$. Furthermore, assuming that the excess oxidative capacity is small, i.e. $r_{p}>\left(r_{o}-a_{1} r_{s}\right) / a_{3}$, one obtains $r_{3}=\left(r_{o}-a_{1} r_{1}\right) / a_{3}$, and thus $r_{3} X=$ $r_{0} X / a_{3}-\left(a_{1} / a_{3}\right)(F / V) S_{i n}$. Using this last expression in (18) gives:

$$
\frac{d P}{d t}=\frac{F}{V}\left(\frac{a_{1}}{a_{3}} S_{i n}+P_{i n}-P\right)-\frac{r_{o} X}{a_{3}}
$$

Similarly, linearization and discretization lead to the following discrete-time model:

$$
P(k h)=\frac{B_{b}\left(q^{-1}\right)}{A\left(q^{-1}\right)}\left[F(k h)-w_{b}(k h)\right]
$$

where $B_{b}\left(q^{-1}\right)=\left(\delta_{b}-\beta_{b} h\right) q^{-1}-\left(\delta_{b}-\right.$ $\left.\beta_{b} h e^{-\alpha h}\right) q^{-2}$, and $w_{b}(k h)=e^{\alpha h} w_{b}(k h-$

$$
\begin{aligned}
& h)+\left(\gamma_{b} / \beta_{b}\right)[X(k h)-X(k h-h)], \text { with } \beta_{b}= \\
& \left(\left(a_{1} / a_{3}\right) S_{i n}+P_{i n}-P_{r e f}\right) / \bar{V}, \gamma_{b}=\bar{r}_{o} / a_{3}, \\
& \delta_{b}=2 \beta_{b}\left(1-e^{-\alpha h}\right) / \alpha-\beta_{b} h .
\end{aligned}
$$

The following averaged linearized discrete-time model will be used:

$$
P(k h)=\frac{B\left(q^{-1}\right)}{A\left(q^{-1}\right)}(F(k h)-w(k h))
$$

where $B=\left(B_{a}+B_{b}\right) / 2$ and $w=\left(w_{a}+w_{b}\right) / 2$.

Though the expressions for $w_{a}$ and $w_{b}$ are different, it is interesting to note that, when $P_{r e f}=P_{i n}$, $w_{a}=w_{b}$. Since the biomass grows exponentially in the first phase and linearly in the second, the disturbance $w$ is unstable. This is a situation for which standard PID-type controllers are inappropriate (Axelsson, 1989). Thus, an adaptive controller based on the internal model principle for disturbance rejection is used here (Valentinotti et al., 2003).

\subsection{Adaptive controller design}

The RST polynomial control law with Q-parameterization is given by (Tsypkin, 1991):

$$
R_{o} F=-S_{o} P+T P_{r e f}+Q(B F-A P)
$$

where $R_{o}, S_{o}$, and $Q$ are polynomials in the backward-shift operator $q^{-1}$. The closed-loop characteristic polynomial is independent of the choice of $Q$ and is given by $A_{c}=A R_{o}+B S_{o}$.

The resulting closed-loop system using the control law (27) is shown in Figure 2. The closed-loop output is given by:

$$
P=\frac{B T}{A_{c}} P_{r e f}-\frac{(R o-Q B)}{A_{c}} w_{B}
$$

with $w_{B}=B w$ a filtered version of the disturbance $w$.

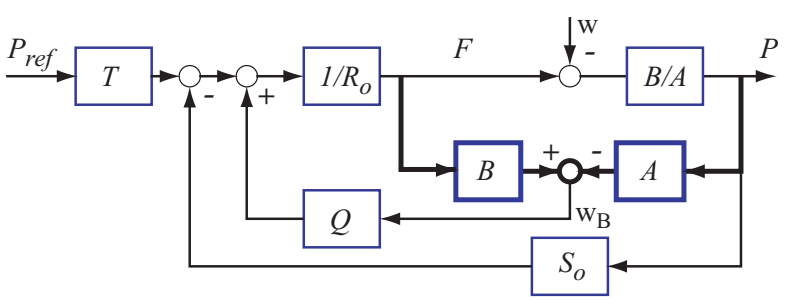

Fig. 2. Block diagram of the controlled system.

The goal of adaptation is to minimize the second term in (28), i.e. the effect of the perturbation $w$, by adjusting $Q$ :

$$
\min _{Q}\left\|\epsilon_{1}-\epsilon_{2} Q\right\|^{2}
$$

where the signals $\epsilon_{1}$ and $\epsilon_{2}$ are defined as $\epsilon_{1}=$ $\frac{R_{o}}{A_{c}} w_{B}$ and $\epsilon_{2}=\frac{B}{A_{c}} w_{B}$. Note that $w_{B}$ can be estimated from the input and output using $\hat{w}_{B}(k h)=$ 
$B\left(q^{-1}\right) F(k h)-A\left(q^{-1}\right) P(k h)$. Equation (29) corresponds to a linear regression problem for the elements of $Q$, for which on-line adaptation can be done using standard algorithms (Ljung, 1987).

\section{SIMULATION RESULTS}

The optimal and the two sub-optimal strategies proposed in Section 4 are implemented in simulation on the model presented in Section 2 using the controller described in Section 5. The substrate concentration and the feed rate for the various strategies are shown in Figures 3 and 4, and the numerical results are given in Table 4 .

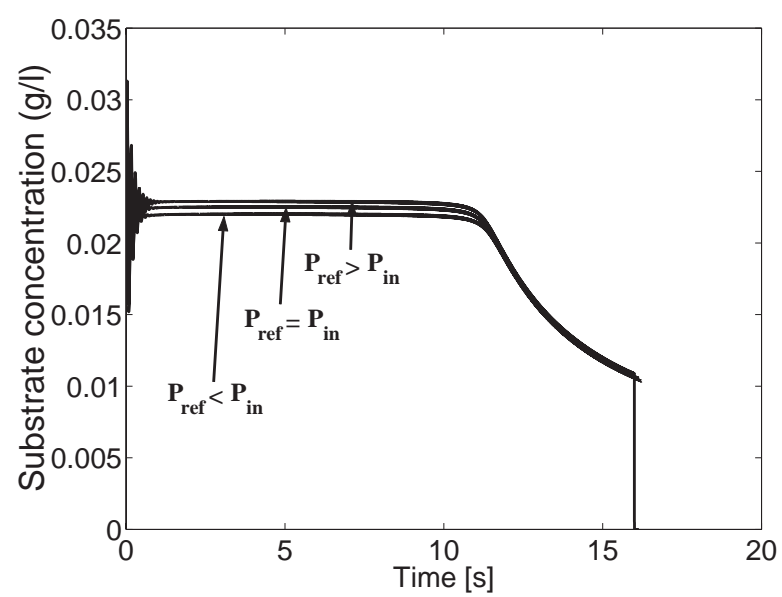

Fig. 3. Substrate concentration profile for various ethanol set points.

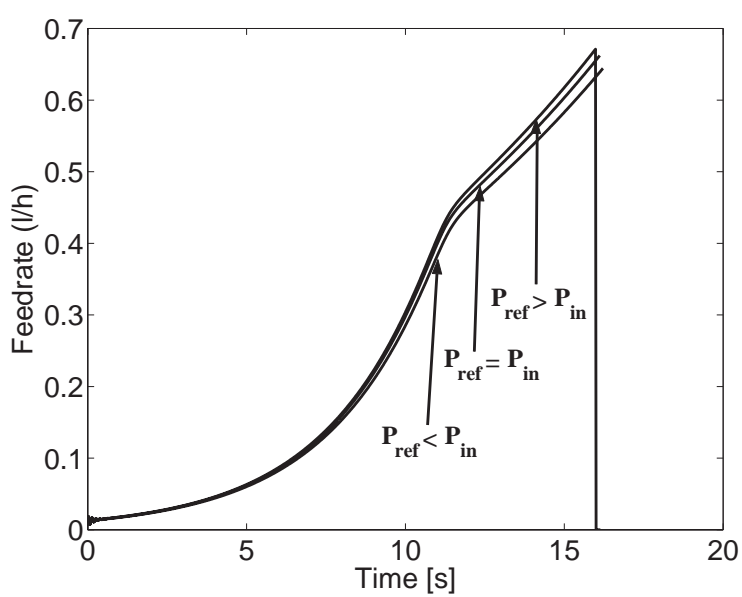

Fig. 4. Feed rate profile for various ethanol set points.

\begin{tabular}{|l|ll|ll|l|}
\hline Strategy & $P_{\text {in }}$ & $P_{\text {ref }}$ & $X\left(t_{f}\right)$ & $V\left(t_{f}\right)$ & $t_{f}$ \\
\hline 1 & 10 & 8 & 74.71 & 7.95 & 16.26 \\
2 & 10 & 10 & 74.25 & 8.00 & 16.12 \\
3 & 10 & 12 & 74.25 & 8.00 & 16.14 \\
\hline
\end{tabular}

Table 4. Optimization results with the various strategies for $(V X)_{d e s}=594 \mathrm{~g}$.

For the optimal Strategy 2, the substrate is always at its critical value $S_{\text {crit }}\left(O_{2}\right)$. Strategies 1 and 3 implement $S<S_{\text {crit }}$ and $S>S_{\text {crit }}$, respectively (see Figure 3).

For the case $P_{r e f}<P_{i n}$, since the bottleneck is not filled with substrate alone, part of the ethanol in the feed is converted to biomass. This way, a slightly higher $X\left(t_{f}\right)$ is obtained. Though the feed stops before the reactor is full, the final time is larger. On the other hand, when $P_{r e f}>P_{i n}$, there is overflow and the reactor is filled slightly faster. However, once the reactor is full, the productivity is less than the desired one. Thus, there is a small batch phase with $F=0$ (see Figure 4 ) so as to produce the required biomass from ethanol.

As seen in Table 4, the minimal time is obtained with Strategy 2. Implementation is by regulating $P(t)$ around $P_{i n}$. Note that no information regarding the model parameters is used in the controller, and the optimal solution is enforced solely from the ethanol measurement through feedback.

\section{CONCLUSIONS}

A non-intuitive but very practical approach for the optimal operation of fed-batch fermentations has been presented. This consists of adding a small amount of product in the feed and maintaining the product concentration in the fermenter constant at its inlet value.

The proposed operating strategy allows maintaining the desired metabolism (either overflow, critical or underflow) even when changes in the value of $S_{\text {crit }}$ occur due to oxygen limitation. In fact, when the oxygen concentration is limiting, regulating $P$ forces the substrate concentration $S$ to decrease in order to match the oxidative capacity of the cells.

Although the analysis and the simulation study were done for $S$. cerevisiae, it is possible to use the proposed approached with other microorganisms presenting an overflow metabolism such as E. coli, a bacteria used for recombinant protein production.

\section{REFERENCES}

Axelsson, J. P. (1989). Modeling and Control of Fermentation Processes. PhD thesis. Lund Institute of Technology.

Chen, L., G. Bastin and V. van Breusegem (1995). A case study of adaptive nonlinear regulation of fed-batch biological reactors. Automatica 31(1), 55-65.

Ljung, L. (1987). System Identification. Theory for the User. Prentice Hall.

Nielsen, J. and J. Villadsen (1994). Bioreaction Engineering Principles. Plenum Press. New York. 
Sonnleitner, B. and O. Käppeli (1986). Growth of Saccharomyces cerevisiae is controlled by its limited respiratory capacity: formulation and verification of a hypothesis . Biotechnology and Bioengineering 28, 927-937.

Tsypkin, Y.Z. (1991). Adaptive-invariant discrete control systems. In: Foundations of Adaptive Control (M. Thoma and A. Wyner, Eds.). Vol. 160 of Lecture Notes in Control and Information Science. pp. 239-268. Springer Verlag.

Valentinotti, S. (2001). Adaptive Rejection of Unstable Disturbances: Application to a Fedbatch Fermentation Process. PhD thesis. Ecole Polytechnique Fédérale de Lausanne.

Valentinotti, S., B. Srinivasan, D. Bonvin, C. Cannizzaro, M. Rhiel and U. von Stockar (2003). Optimal operation of fed-batch fermentations via adaptive control of overflow metabolite. Control Engineering Practice 11(6), 665-674.

van Hoek, P., J. van Dijken and J. Pronk (1998). Effect of specific growth rate on fermentative capacity of baker's yeast. Applied and Environmental Microbiology 64(11), 4226-4233. 